\title{
Uterus myomatosus - Diagnostik und Therapie insbesondere bei Kinderwunsch
}

Felix Neis, Christoph Oettling, Katrin Neis

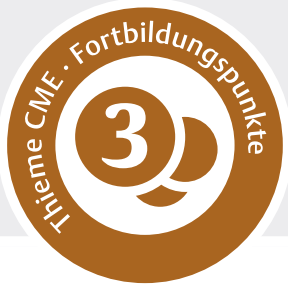

Unter den bei Frauen im gebärfähigen Alter mit Inzidenzen von 20-30\% relativ häufigen Myomen können vor allem die submukösen und intramuralen Subtypen die Fertilität beeinträchtigen. Bei Kinderwunsch bestehen jedoch einige Therapieverfahren, die vor einer angestrebten Konzeption angewandt werden können und gute und erfolgreiche Schwangerschaftsverläufe erlauben.

\section{Inzidenz}

Der Altersdurchschnitt von Frauen mit Kinderwunsch ist in den letzten Jahren in Deutschland stetig angestiegen. Eine Studie aus dem Jahr 2015 zeigt, dass bei über 50\% aller Frauen in Deutschland im Laufe ihres Lebens Myome diagnostiziert werden [1]. Da es sich bei Myomen um hormonabhängige Tumoren des Uterus handelt, verwundert es nicht, dass die Anzahl der Myome bis zum Erreichen der Postmenopause ansteigt und anschließend wieder abfällt. Im gebärfähigen Alter sind ca. 20-30\% aller Frauen von Myomen betroffen. Hauptsächlich erhält jedoch die Altersgruppe der 46- bis 50-jährigen Frauen mit knapp 63\% die Diagnose „Uterus myomatosus“. Insbesondere im Hinblick auf den immer später auftretenden Kinderwunsch wird klar, dass es sich hierbei um ein relevantes Problem handelt.

Merke

Etwa 20-30\% aller Frauen im gebärfähigen Alter sind von Myomen betroffen.

Bei einer Befragung von 1756 Frauen aus Westeuropa kam E. Downes zu der Feststellung, dass bei 9-32,5\% der Frauen bis zur Diagnosestellung „Myom“ mehr als 5 Jahre vergingen [2].

Bei einem Myomwachstum in der Postmenopause oder bei sehr schnellem Myomwachstum muss als Differenzialdiagnose eine maligne Erkrankung im Sinne eines Sarkoms in Betracht gezogen werden.

\section{Entstehung von Myomen}

Myome sind gutartige, hormonsensitive Tumoren der glatten Muskulatur des Uterus. Histologisch zeichnen sie sich durch einen hohen Anteil an extrazellulärer Matrix (Kollagen, Proteoglykan, Fibronektin) aus, welche von einer dünnen Pseudokapsel umgeben ist. Sie entwickeln sich monoklonal, das heißt aus nur einer Muskelzelle.

Ursächliche Faktoren, die zur Entstehung und zum Wachstum von Myomen beitragen, sind noch weitestgehend ungeklärt. Bekannt ist jedoch die Tatsache, dass Myome sowohl unter Östrogen- als auch unter Progesteroneinfluss an Volumen zunehmen. Eine frühe Menarche scheint das Risiko für Myome zu erhöhen, wohingegen eine hohe Parität eher zu schützen scheint [3]. Die Rolle von Steroiden, Zytokinen, Wachstumsfaktoren, microRNA und weiteren Faktoren wird zurzeit in der Literatur diskutiert [4].

Auch genetische Faktoren und die ethnische Zugehörigkeit scheinen einen Einfluss auf die Entstehung von Myomen zu haben. So sind dunkelhäutige Frauen eher prädisponiert für das Auftreten von Myomen als Frauen mit weißer Hautfarbe oder Asiatinnen. Auch bei positiver Familienanamnese lässt sich eine Häufung beobachten.

Ebenso lassen sich Auswirkungen der Lebensführung auf Auftreten und Wachstum von Myomen beobachten. So lassen eine regelmäßige sportliche Aktivität und das Einhalten einer Diät bei Übergewicht eine Verlangsamung des Myomwachstums vermuten, wohingegen VitaminD-Mangel, Rauchen, Übergewicht und die Einnahme von Hormonpräparaten das Wachstum zu beschleunigen scheinen.

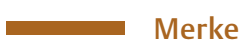

Die Entstehung von Myomen ist multifaktoriell beeinflusst, u.a. hormonell, genetisch und durch Umweltfaktoren oder den Lebensstil. 


\section{Symptome}

Die meisten Myome sind symptomlos. Sie sind meist ein Zufallsbefund im Rahmen einer Routineuntersuchung. Werden Myome symptomatisch, so können sie meist durch die bimanuelle Untersuchung oder sonografisch diagnostiziert werden.

Die durch Myome hervorgerufenen Beschwerden können sich sehr unterschiedlich manifestieren. Die auftretenden Symptome sind Ausdruck der Lage (submukös, intramural oder subserös) sowie auch der Größe des jeweiligen Myoms.

Die Hauptsymptome sind:

- Blutungsstörungen

- Hypermenorrhö, auch mit sekundärer Anämie $(\mathrm{Hb}<12 \mathrm{~g} / \mathrm{dl})$

- Menorrhagie

- Metrorrhagie

- Dysmenorrhö

- Unterleibsschmerzen, Druckgefühl durch Verdrängung anderer Organe (Blase, Darm, Ureter)

- Pollakisurie

- Obstipation

- Fertilitätsprobleme

\section{Merke}

Aufgrund der Heterogenität der Myome treten häufig multiple Symptome in unterschiedlicher Ausprägung auf.

\section{Einteilung der Myome}

Die Gliederung von Myomen erfolgt anhand der FIGOKlassifikation aus dem Jahr 2011 ( $\triangleright$ Tab. 1, Abb. 1) [5]. Hiermit lassen sich reproduzierbare Befunde erstellen. Die Typen 0 bis 1 beschreiben Myome, die direkt unterhalb des Endometriums liegen. Das Typ-2-Myom liegt submukös und mehr als 50\% im Myometrium. Typ 3 und
4 liegen komplett intramural, wobei Typ 3 noch Kontakt zum Endometrium hat, das Uteruskavum aber nicht imprimiert. Typ 5, Typ 6 und Typ 7 sind subseröse Myome ( $\triangleright$ Abb. 2, Abb. 3). Beim Typ 5 liegen mehr als $50 \%$ intramural, beim Typ 6 weniger als $50 \%$ intramural. Gestielte Myome werden als Typ 7 bezeichnet. Bei der Gruppe 2-5 handelt es sich um ein transmurales Myom, also ein Myom, welches durch die gesamte Gebärmutterwand reicht. Parasitäre oder intraligamentäre Myome werden als Typ 8 bezeichnet.

\section{Diagnostik}

Der Untersuchungsgang bei Patientinnen mit Myomen bzw. die Diagnosesicherung bedarf meist keiner gesonderten Diagnostik. Hauptbestandteile sind die gynäkologische Anamnese und die bimanuelle Tastuntersuchung. Ebenso sollte stets eine Inspektion des äußeren Genitales und des Abdomens sowie die vaginale Einstellung der Portio mittels Spekula erfolgen. Im Rahmen der Palpation können Größe und Beweglichkeit des Uterus bestimmt sowie meist die Lage der Myome erfasst werden. In der Regel tastet sich ein glatt begrenzter, gut mobiler, derber Befund. Insbesondere bei sehr großen Uteri ist eine Größenangabe mittels „Querfinger (QF) über der Symphyse oder unterhalb/oberhalb des Nabels“ sinnvoll. Ebenfalls ist auf die Untersuchung des Septum rectovaginale zu achten, um eine eventuell zusätzlich bestehende Endometriose diagnostizieren zu können.

\section{PRAXISTIPP}

Die bildliche Darstellung der Myome sollte standardmäßig per Vaginalsonografie erfolgen ( $\bullet$ Abb. 4). Bei sehr großen Myomen/Uteri kann zusätzlich die abdominale sonografische Darstellung hilfreich sein. Ein Harnstau sollte vor allem bei großen Myomen mittels Nierensonografie ausgeschlossen werden.

\Tab. 1 FIGO-Klassifikation der Myome (Daten nach [5]).

\begin{tabular}{|l|l|l|}
\hline Klassifikation & Typ & Beschreibung \\
\hline submukös & 0 & unter dem Endometrium, gestielt in der Gebärmutterhöhle \\
\hline \multirow{2}{*}{ intramural } & 1 & unter dem Endometrium, $<50 \%$ im Myometrium \\
\hline & 2 & unter dem Endometrium, $>50 \%$ im Myometrium \\
\hline 3 & $100 \%$ in der Muskulatur, Kontakt zum Endometrium \\
\hline 4 & im Myometrium \\
\hline 5 & subserös, $>50 \%$ im Myometrium \\
\hline subserös & $2-5$ & geht durch die gesamte Gebärmutterwand, transmural \\
\hline & 6 & subserös, < $50 \%$ im Myometrium \\
\hline & 7 & gestielt, subserös \\
\hline & 8 & andere (intraligamentär, parasitär, etc.) \\
\hline
\end{tabular}




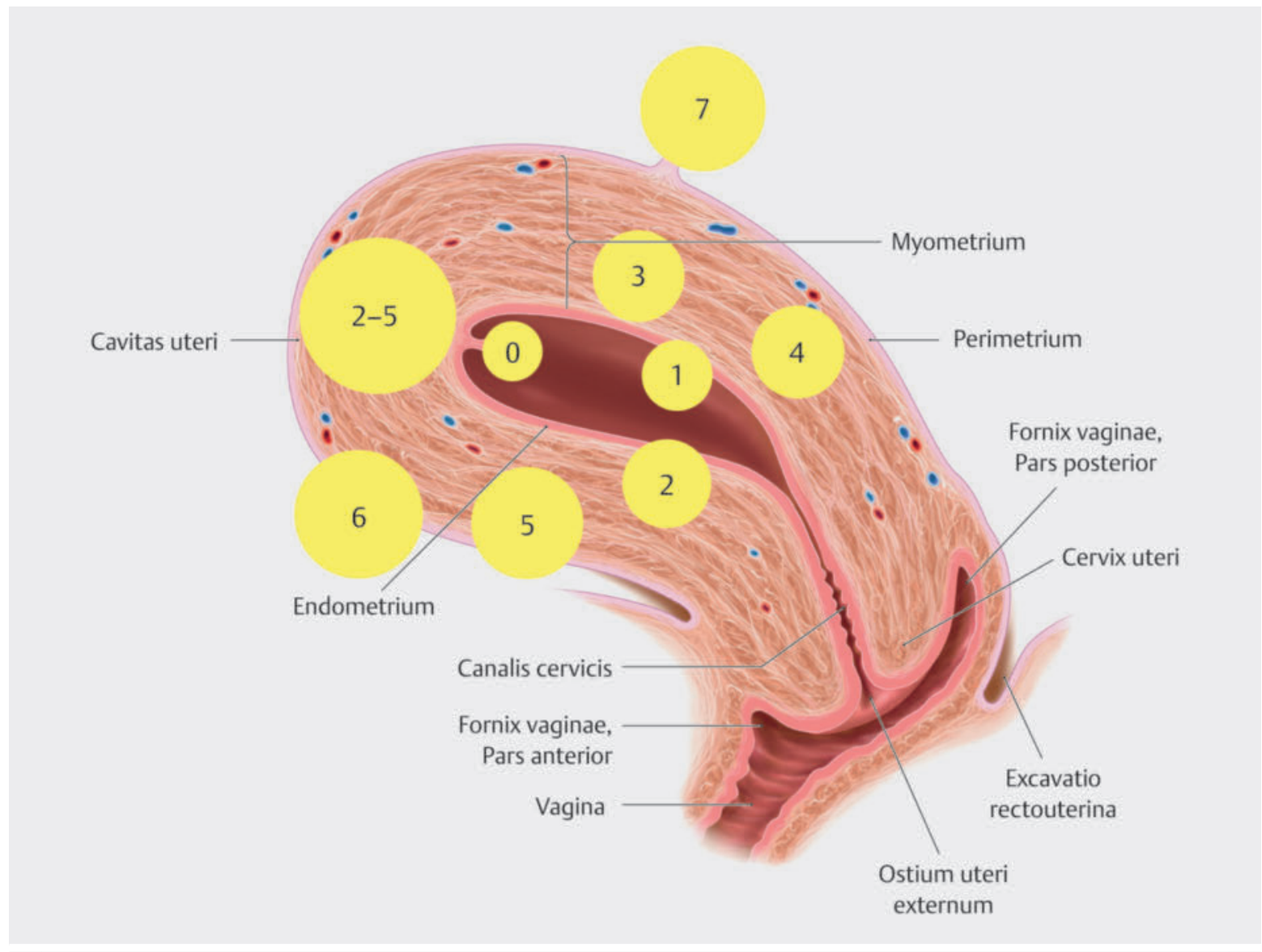

Abb. 1 FIGO-Klassifikation der Myome (Daten nach [5]). Quelle: Schünke M, Schulte E, Schumacher U et al., Hrsg. Prometheus LernAtlas - Innere Organe. Illustrationen von Voll M und Wesker K. 5. Auflage. Stuttgart: Thieme; 2018.

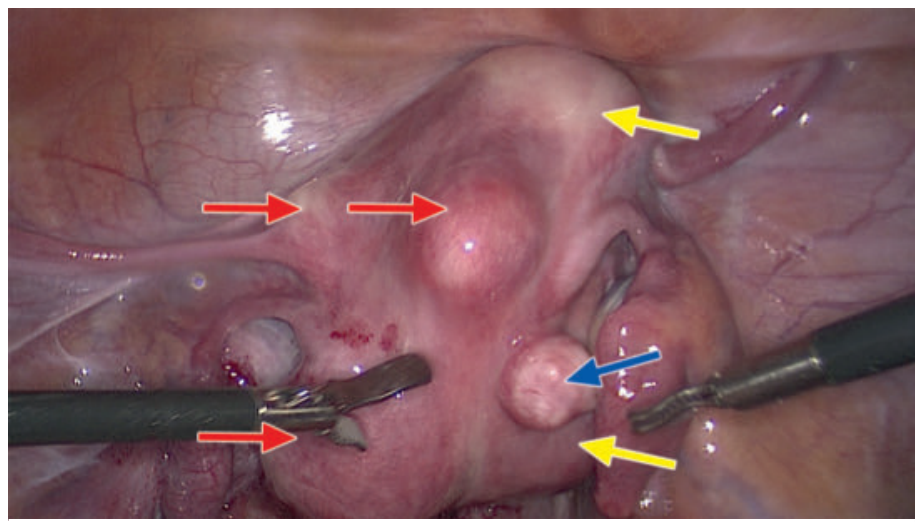

Abb. 2 Laparoskopische Sicht auf einen Uterus myomatosus mit Myomen aller Typen. Gelb: Typ 2-5; Rot: Typ 6; Blau: Typ 7. 


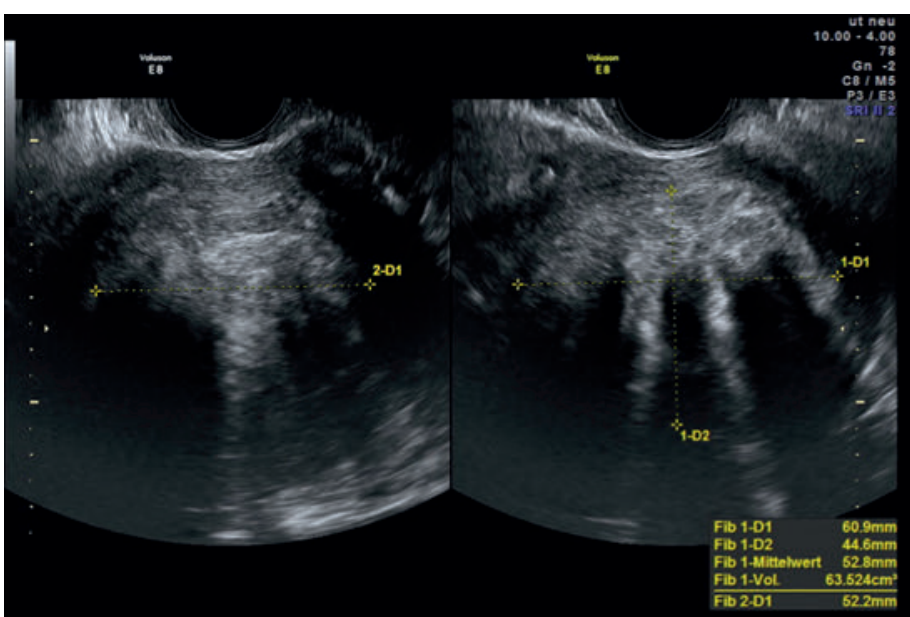

- Abb. 4 Transvaginalsonografische Darstellung eines Typ-2-5-Myoms der Uterushinterwand. Quelle: Dr. med. Felix Neis/Thieme Gyn Community.

Die Einteilung der Myome erfolgt mithilfe der FIGO-Klassifikation anhand ihrer anatomischen Lokalisation (Vorderwand, Hinterwand, Fundus, etc.) ( $\bullet$ Abb. 1). Eine Größenangabe in mindestens 2 Ebenen sollte vorgenommen werden.

\section{PRAXISTIPP}

Durch die konsequente Beschreibung der Myome analog der FIGO-Klassifikation mit Lagebeschreibung und Größenangabe in mindestens zwei Ebenen gelingt eine gute Reproduzierbarkeit der Untersuchungsbefunde.

Zur Verbesserung der Darstellbarkeit der submukösen Myome kann eine Hydrosonografie durchgeführt werden. Hierfür werden mit einem kleinen Katheter ca. 10$20 \mathrm{ml}$ physiologische Kochsalzlösung nach Desinfektion der Vagina in das Uteruskavum eingebracht. Hierdurch können intrakavitäre Befunde besser bezüglich ihrer myometranen Lokalisation dargestellt werden.

Bei sehr ausgedehntem Myombefall kann die Durchführung einer MRT oder CT hilfreich sein. Insbesondere bei Verdacht auf ein malignes Geschehen ist dies anzuraten. Bisher existieren keine Kriterien, mit denen sich ein Sarkom klinisch oder bildgebend $100 \%$ ig beweisen oder ausschließen lässt. Auch gibt es keine spezifischen Symptome, die auf ein Sarkom schließen lassen. Hinweise können ein schnelles Myomwachstum, eine gute Perfusion und ein höheres Patientenalter sein. Die höchste Inzidenz liegt zwischen dem 45. und 59. Lebensjahr [6]. Somit ist das Risiko eines Sarkoms bei Patientinnen mit aktuellem Kinderwunsch eher gering.
Cave

Eine MRT- oder CT-Bildgebung kann das Vorhandensein eines Sarkoms vermuten, aber nicht definitiv ausschließen.

Myomspezifische Laboruntersuchungen existieren nicht. Je nach Symptomatik sind jedoch Blutuntersuchungen sinnvoll. Bei starken Blutungsstörungen kann für die Planung der Therapie sowie den Zeitpunkt der Therapie die Bestimmung des Hämoglobins $(\mathrm{Hb})$ hilfreich sein. Ein niedriger $\mathrm{Hb}$-Wert kann so zu einem früheren Operationstermin oder der präoperativen Gabe von Erythrozytenkonzentraten führen.

Bei großen Myomen kann es durch Druck auf die Beckenwände zu einem Harnstau kommen. In diesen Fällen sollte zum Ausschluss einer Nierenschädigung das Kreatinin bestimmt werden.

\section{PRAXISTIPP}

Eine Zeichnung während der Aufklärung und Beratung kann der Patientin helfen, die Lokalisation und Größe der Myome sowie die damit verbundenen Symptome und Auswirkungen auf die Fertilität besser zu verstehen.

\section{Auswirkung auf die Fertilität}

Literatur zu Auswirkungen von Myomen und ihrer jeweiligen Therapie auf die Fertilität ist nur als retrospektive Studien und zum Teil mit nur kleinen Kollektiven verfügbar [7]. Verständlich ist jedoch, dass eine Veränderung des Uteruskavums, bedingt durch submuköse Myome oder Impression von intramuralen Myomen, die Fertilität negativ beeinträchtigt. Subseröse Myome scheinen die Fertilität nicht zu beeinflussen [8]. Abhängig sind diese Auswirkungen natürlich immer auch von der Größe der Myome.

Bei der Analyse von Frauen mit Myomen aller Lokalisationen fallen signifikant erniedrigte Chancen für eine klinische Schwangerschaft, Implantation, Fortbestehen einer Schwangerschaft und Lebendgeburten im Vergleich zu Frauen ohne Myome auf. Auch ist die Spontanabortrate bei Frauen mit Myomen signifikant erhöht. Die Effekte sind bei Kavumbeteiligung schwerwiegender [8].

\section{Merke}

Insbesondere bei einer Veränderung des Uteruskavums kommt es zu verschlechterten Fertilitätschancen. 
Wegen der großen Varianz in Bezug auf Lage, Anzahl und Größe ist der exakte Einfluss des jeweiligen Myoms auf die Fertilität nur schwierig zu beziffern. Ein $2-3 \mathrm{~cm}$ großes subseröses Myom mag eine Schwangerschaft kaum stören, ein 2-3 cm großes submuköses Myom hingegen mit einer hohen Wahrscheinlichkeit. Deshalb erweist sich die Studienplanung bezüglich des Einflusses eines spezifischen Myomtypus als extrem schwierig.

Einige Studien zeigen einen Einfluss von Myomen auf die Fertilität ab einer Größe $>2,85 \mathrm{~cm}$ oder $>3 \mathrm{~cm}$, andere Studien zeigen einen negativen Einfluss erst ab einer Myomgröße $>5 \mathrm{~cm}$ [9].

\section{Veränderung der Myome in der Schwangerschaft}

Da Myome hormonsensible Tumoren sind, können diese auch während der Schwangerschaft wachsen. Es handelt sich hierbei typischerweise um ein triphasisches Wachstum, wenngleich dieses nicht linear zu sein scheint [10]:
1. Trimenon:
signifikantes Wachstum
2. Trimenon:
verlangsamtes Wachstum bis Größenstabilisierung
2.-3. Trimenon: eher Größenreduktion (Trend)
Wochenbett:
Größenreduktion bis -stabilisation

Bei bis zu 70\% der Patientinnen mit Myomen verläuft die Schwangerschaft unauffällig. Lediglich bei ca. $10 \%$ wird der Schwangerschaftsverlauf durch die vorliegenden Myome negativ beeinflusst. Hiervon ist bei ca. 10-30\% mit schweren unerwünschten Ereignissen zu rechnen.

\section{Therapiestrategien}

Die Therapiemöglichkeiten der Myome sind sehr vielschichtig - genauso wie die Lage und Größe der Myome. Ziel einer guten Arzt-Patienten-Beratung ist es immer, die Patientin auf den Wissensstand zu bringen, mit dem sie, ggf. auch mit dem Partner gemeinsam, in der Lage ist die Therapieentscheidung im sogenannten „Informed Consent" zu treffen.

Je nach Lebenssituation, Aktualität des Kinderwunsches, Myomcharakteristika, Alter und jeweiligem Patientenwunsch muss die passende Therapie gemeinsam gefunden werden.

Generell gilt, dass eine Operation oder Intervention nur dann durchgeführt werden sollte, wenn zu erwarten ist, dass ein aktueller oder prospektiver Kinderwunsch gefährdet ist oder die Patientin Symptome hat.
Infrage kommen medikamentöse, interventionelle und operative Therapiestrategien:

- operativ

- hysteroskopische Myomresektion

- laparoskopische Myomenukleation

- abdominale Myomenukleation

- laparoskopische Radiofrequenzablation (Acessa ${ }^{\circledR}$ )

- transzervikale Radiofrequenzablation (Sonata ${ }^{\circledR}$ )

- interventionell

- MRT-gesteuerter fokussierter Ultraschall (MRgFUS)

- Embolisation der A. uterina (UAE)

- medikamentös

- GnRH-Analoga

- Ulipristalacetat (Esmya)

\section{Operative Therapie bei Kinderwunsch}

Hysteroskopische Myomresektion

Indikation zur operativen Hysteroskopie sind Myome mit Lage im Uteruskavum (Typ 0, Typ 1 und Typ 2). Mit Einschränkungen sind unter Umständen auch Myome Typ 3 per operativer Hysteroskopie resezierbar. Da diese per Definition das Uteruskavum jedoch nicht imprimieren, ist das Auffinden der Myome deutlich erschwert und gelingt meist nur per simultanem transabdominalen oder rektalen Ultraschall.

Die operative Hysteroskopie sollte aus Gründen der besseren intrakavitären Sicht und zur Vermeidung der Störung einer laborchemisch eventuell noch nicht nachweisbaren Frühschwangerschaft in der ersten Zyklushälfte erfolgen.

\section{Die Hysteroskopie sollte in der ersten Zyklushälfte erfolgen.}

Bei der operativen Hysteroskopie kann man wählen zwischen den konventionellen Verfahren zur monopolaren und bipolaren Resektion und den mechanischen (Stanze vs. Shaver) Verfahren zur Entfernung der Myome. Die Myome werden jeweils unter Sicht aus dem Cavum uteri entfernt.

\footnotetext{
Merke

Ziel ist es in jedem Fall, die intrakavitär sichtbaren Myome zu entfernen und die Form des Kavums wiederherzustellen.
}

Bei der Entfernung von Myomen mit einem mechanischen Instrument (Shaver, Stanze) saugt man das Myom an die resezierende Spitze an und reseziert es dann schrittweise ( $\triangleright$ Abb. 5). Mit den mechanischen Verfahren gelingt es lediglich, die in das Kavum reichenden Myome zu resezieren. Tief transmurale Anteile sind die Domäne der konventionellen operativen Hysteroskopie, so z. B. bei einem Typ-2-Myom oder bei festen Myomen. 


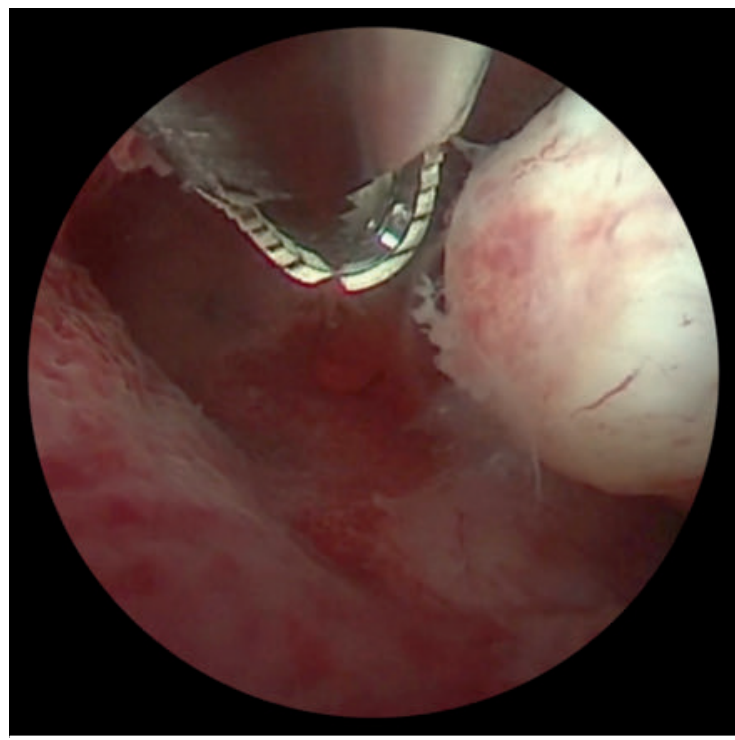

- Abb. 5 Hysteroskopischer Shaver zur Resektion des Typ-0-Seitenwandmyoms links. Quelle: Thieme GynCommunity.

\section{PRAXISTIPP}

Die Entfernung von sehr harten Myomen erweist sich als sehr schwierig, da diese Myome nur schlecht in das mechanische Instrument eingezogen werden können. Die zum Teil sehr kleinen Fragmente sind in der pathologischen Aufarbeitung meist sehr schwierig zu beurteilen.

Die operative (mono- und bipolare) Hysteroskopie ermöglicht die genaue Resektion des Myoms bis zum Übergang in das Myometrium. So kann der pathologische Befund entfernt werden, ohne das Myometrium zu schädigen. Dies gelingt deshalb, weil Myome verdrängend und nicht invasiv in das Myometrium hineinwachsen. Den Übergang zum gesunden Myometrium erkennt man durch eine Änderung der Struktur und des Gewebes. Die Myome stellen sich wirbelig, weiß- bis gelblich mit derber Struktur dar. Das Myometrium erscheint hingegen rötlich ( $\triangleright$ Abb. 6). Beim Schnitt durch das Myometrium entsteht eine leicht geriffelte Schnittfläche.

Mit der elektrischen Hochfrequenzschlinge lassen sich problemlos Myome bis $2 \mathrm{~cm}$ in einer Sitzung entfernen. Myome bis zu einer Größe von ca. $4 \mathrm{~cm}$ können von erfahrenen Operateuren in einer Sitzung saniert werden. Je größer das Myom und je tiefer die intramurale Lage, umso schwieriger ist die Resektion.

Technikbedingt gibt es Risiken wie das Transurethrale Resektions-(TUR-)Syndrom oder eine Hyperhydratation der Patientin durch Übertritt der verwendeten Distensions-

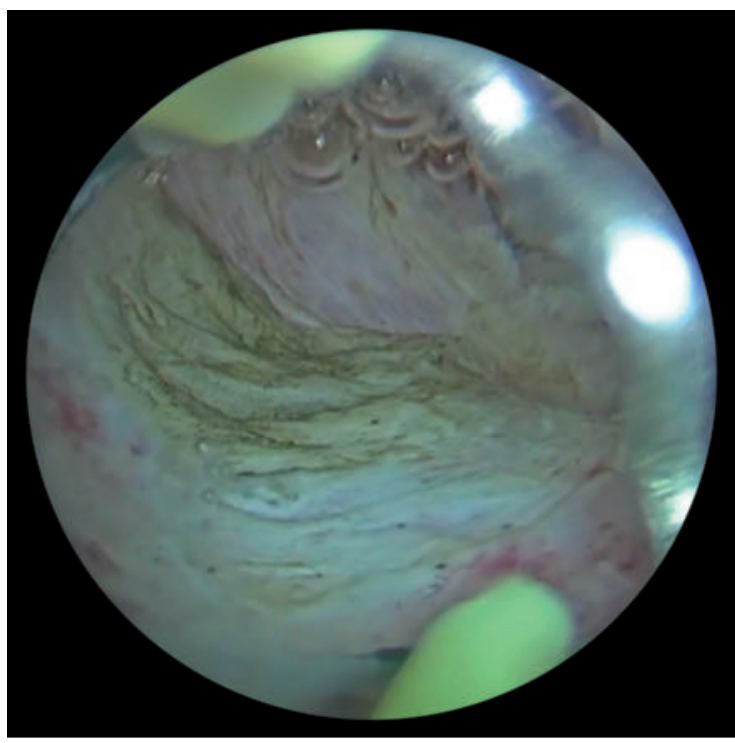

- Abb. 6 Hysteroskopische Resektion des Myoms an der Hinterwand. Das Myom stellt sich weißlich, wirbelig dar. Direkt dahinter liegt das unauffällige, leicht rosafarbene Myometrium. Quelle: Thieme Gyn-Community.

lösung (elektrolytfrei bei monopolarem Strom; elektrolythaltig bei bipolarem Strom) in den Blutkreislauf der Patientin. Je größer und je tiefer das Myom intramural liegt, umso höher ist das Risiko für ein TUR-Syndrom/Hyperhydratation. Zwingend ist deshalb ein kontinuierliches Monitoring der Ein- und Ausfuhr der verwendeten Flüssigkeit.

\section{PRAXISTIPP}

Bei Verwendung von Purisolen (elektrolytfrei) sollte bei einem Defizit von $1000 \mathrm{ml}$ und bei Kochsalzlösung ( $\mathrm{NaCl}$, elektrolythaltig) ab einem Defizit von $2000 \mathrm{ml}$ die Operation sicherheitshalber abgebrochen werden.

Ist eine 2. Sitzung notwendig, so sollte diese im Abstand von 6-8 Wochen erfolgen. Über dieses technikbedingte Risiko der zweizeitigen Operation muss die Patientin präoperativ aufgeklärt werden.

Gasblasen, die durch die Resektion oder das wiederholte Einführen des Hysteroskops entstehen, können in seltenen Fällen zu einer Luftembolie führen. Deshalb ist stets auf das Entfernen der Luftblasen aus dem Kavum zu achten. Dies gelingt durch Öffnen des Ablaufschlauches, welcher deckenwärts gerichtet sein muss.

Merke

Die Wiederherstellung des Uteruskavums verbessert die Nidation und verringert das Abortrisiko. 
Die Vermeidung von postoperativen Adhäsionen durch Östrogenisierung oder durch Verwendung von flüssigen oder gelartigen Adhäsionsprophylaktika erscheint nach aktuellster Literatur in den meisten Fällen nicht notwendig, kann aber bei sehr ausgedehnten Befunden erwogen werden.

Eine postoperative sichere Antikonzeption ist meist nur für 2-3 Monate notwendig. Bei Eintritt einer Schwangerschaft kann in der Regel ein Spontanpartus angestrebt werden.

\section{Laparoskopische Myomenukleation}

Der Goldstandard zur Entfernung aller intramuralen und subserösen Myome ist die laparoskopische Myomenukleation. Sie bietet die Vorteile des minimalinvasiven Zugangsweges mit schneller postoperativer körperlicher Erholung, schneller Wiederaufnahme der Arbeitsfähigkeit, kurzem Krankenhausaufenthalt, geringen Schmerzen und guten kosmetischen Ergebnissen.

Die laparoskopische Myomenukleation sollte immer mit einer diagnostischen Hysteroskopie kombiniert werden. Die Anzahl und Positionierung der Einstiche orientiert sich an der Größe des Uterus und der Lage der Myome. Zur besseren Darstellbarkeit der Anatomie empfiehlt es sich, einen Uterusmanipulator zu verwenden, über den vor und nach Myomenukleation eine Chromopertubation durchgeführt wird.

\section{Merke \\ Für intramurale Myome ist der Goldstandard die la- paroskopische Myomenukleation.}

Erfahrene Operateure können Myome bis zu 10 cm Größe laparoskopisch enukleieren. Mit steigender Anzahl der Myome erhöht sich der Schwierigkeitsgrad der Operation. Oft sind hierfür mehrere Inzisionen des Uterus nötig. Auf eine ausreichende Distanz zu den Tuben sollte geachtet werden, um diese bei der nachfolgenden Naht nicht zu okkludieren.

Im Falle einer Eröffnung des Cavum uteri mit notwendiger Rekonstruktion per Naht sollte diese mehrschichtig erfolgen. Grundvoraussetzung für die laparoskopische Myomenukleation ist daher eine entsprechende Expertise und notwendige Erfahrung des Operateurs in laparoskopischer Nahttechnik. Kann eine mehrschichtige Naht aus technischen Gründen nicht durchgeführt werden, profitiert die Patientin in diesen Fällen von einer Naht per (Mini-)Laparotomie (s. auch $>$ Abb. 10).

Die Enukleation des Myoms erfolgt nach Eröffnung der Serosa durch Zug und Gegenzug am Myom. Schrittweise wird das Myom unter Koagulation der Myomkapsel aus dem gesunden Myometrium geschält. Die so entstehen-

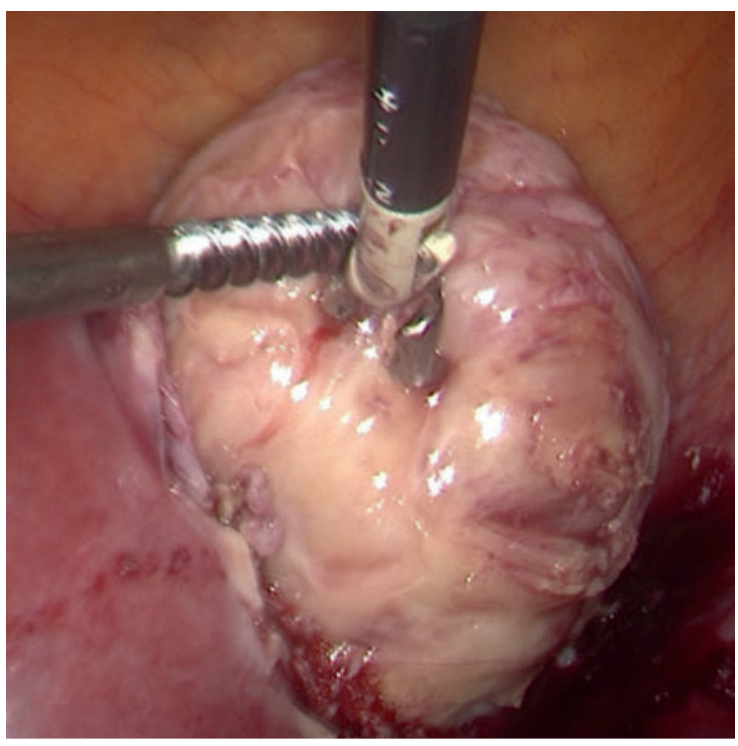

Abb. 7 Laparoskopische Myomenukleation eines Seitenwandmyoms vom Typ 2-5 mithilfe eines Myombohrers.

de Wundfläche entspricht dem Myomumfang. Scharfe Fasszangen und ein Myombohrer sind hier hilfreiche Instrumente, um das Myom aus dem Uterus zu entfernen ( $\triangleright$ Abb. 7).

Bezüglich der Blutungskontrolle kann eine subkapsuläre Injektion von Kochsalzlösung mit oder ohne vasokonstriktive Substanzen (Vasopressin, Epinephrin, Tranexamsäure etc.) oder ein Setzen von laparoskopischen Klemmen an $\operatorname{der} A$. uterina oder A. ovarica erfolgen [11].

Mit dem Ziel, eine möglichst optimale Wundheilung zu erreichen, sollte auf ausgedehnte Koagulation verzichtet werden, um das Gewebe bestmöglich zu schonen. Mit einer blutstillenden, komprimierenden, ggf. mehrschichtigen Adaptation der Wundränder werden Hämatome vermieden. Zu achten ist auf eine suffiziente Naht ohne Nekrosen, um die Stabilität des Myometriums bei einer nachfolgenden Schwangerschaft bestmöglich zu erreichen ( $\triangleright$ Abb. 8). Die Wahl der Nahttechnik (Einzelknopfnaht vs. fortlaufende Naht) sowie des Fadens (geflochten vs. monofilament) obliegt dem Operateur.

Um postoperative Adhäsionen zu vermeiden, sollte die Enukleation der Myome über so wenige Inzisionen wie möglich erfolgen. Zudem ist darauf zu achten, dass so wenig Fadenmaterial wie möglich an der Außenseite des Uterus verbleibt.

Cave

Das höchste Risiko für Adhäsionen besteht bei einer Myomenukleation an der Uterushinterwand. 


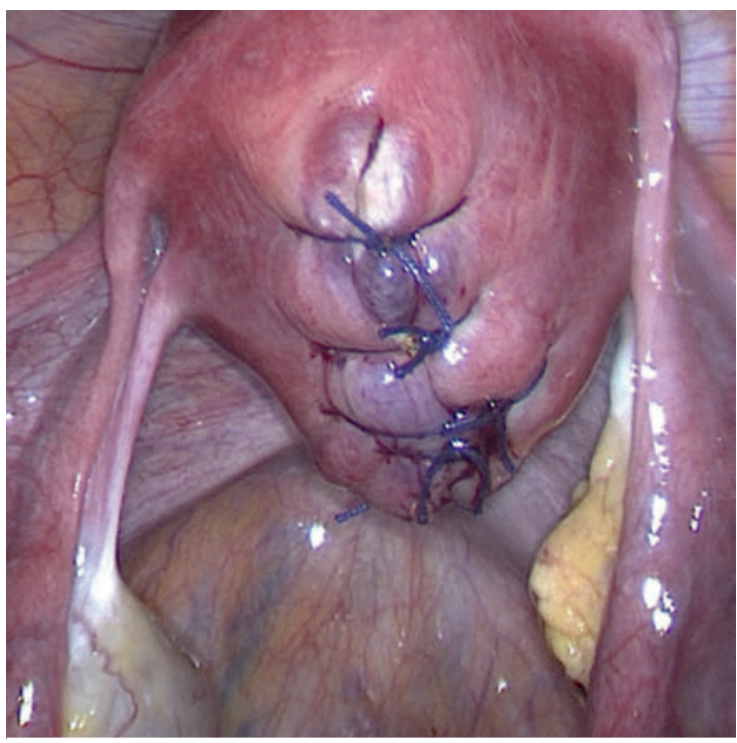

- Abb. 8 Naht nach laparoskopischer Myomenukleation eines Hinterwandmyoms vom Typ 2-5 mit Vicryl-0Einzelknopfnähten.

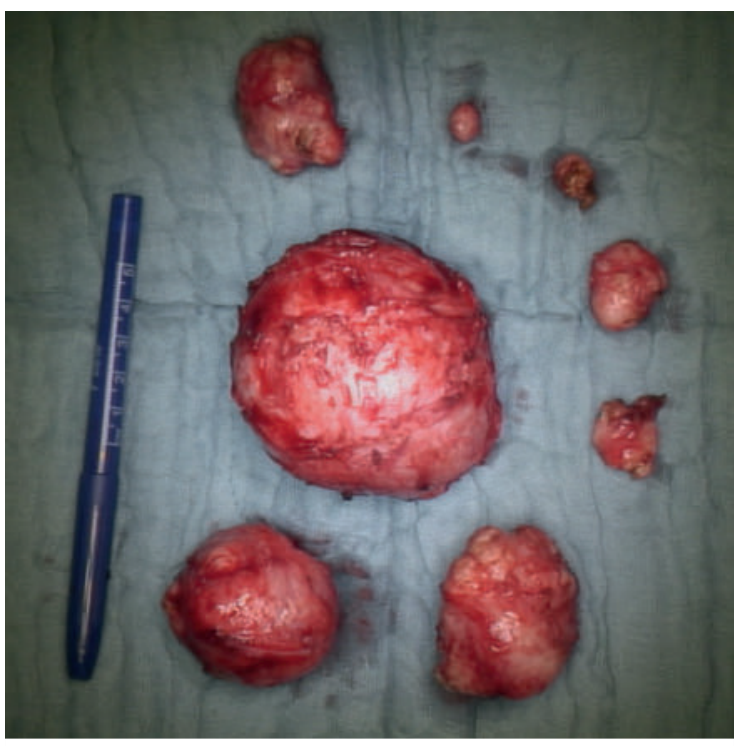

- Abb. 9 Postoperative Fotodokumentation von 8 bis zu $7 \mathrm{~cm}$ großen Myomen, welche per abdominaler Myomenukleation entfernt wurden. Stift auf der linken Seite zum Größenvergleich.

\section{Abdominale Myomenukleation}

Bei ausgedehnten Befunden (Myomgröße $>10 \mathrm{~cm}$, zahlreiche Myome) und anatomisch diffiziler Lage (tief intra-(transmural) ist der abdominale Zugangsweg zu bevorzugen.

Hierfür ist eine präoperative Planung der Schnittführung und Beratung der Patientin durch den Operateur notwendig. Kosmetisch ansprechendere Ergebnisse werden durch eine Querlaparotomie erreicht. Eine Längseröffnung der Faszie kann den Zugang in das Operationsgebiet bei großen Myomen oder ungünstiger Lage erweitern. Der Vorteil der primären Längslaparotomie liegt in der Möglichkeit zur Erweiterung der Schnittführung bis unter das Xiphoid.

\section{Cave}

Eine Konversion von einer Querlaparotomie auf eine Längslaparotomie ist unbedingt zu vermeiden.

Der Vorteil des abdominellen Zugangs liegt in der Möglichkeit, den Uterus zu palpieren und so auch kleine Myome, welche im laparoskopischen Zugang unberücksichtigt bleiben, mitzuentfernen ( $\boldsymbol{A} \mathbf{A b b}$. 9). Ob es sich bei diesen kleinen Myomen um schwangerschaftsrelevante Myome handelt oder diese aufgrund der Größe und Lage unbedenklich sind, ist aus der vorliegenden Literatur nicht abschließend zu klären.

\section{Rezidive}

Bei jeder präoperativen Aufklärung muss das Risiko von Rezidiven angesprochen werden. Das Risiko steigt, je mehr Myome vorhanden sind und je jünger die Patientin ist. Zudem unterliegt es je nach operativem Zugangsweg erheblichen Schwankungen.

Nach abdominalen Myomenukleationen werden Rezidivquoten von 15,4-62\% nach 5 Jahren beschrieben. Die Rezidivquoten nach laparoskopischer Myomenukleation liegen bei $52,9-62,1 \%$ nach 5 Jahren und $84,8 \%$ nach 8 Jahren [12]. Die jedoch ebenfalls beschriebene niedrige ReOperations-Rate von 2,4-6,7\% nach 5 Jahren und 16\% nach 8 Jahren, spricht für die Theorie, dass es sich bei diesen Myomen nicht um schwangerschaftsrelevante Myome handelt.

\section{Merke}

Die Rezidivquote nach Myomenukleation liegt bei bis zu $62 \%$ nach 5 Jahren.

\section{Uterusruptur}

Eine der Hauptsorgen nach Myomenukleation stellt die Uterusruptur dar, wenngleich das Risiko mit $0,7-1 \%$ sehr niedrig ist [12]. Risikofaktoren sind Gewebeschädigungen (z.B. durch ausgedehnte Koagulation), Infektionen oder Hämatome im Bereich des Wundhöhle. Bei einschichtiger Rekonstruktion konnten Studien ein 4-fach erhöhtes Risiko für Dehiszenzen im Vergleich zu zweischichtiger Rekonstruktion zeigen. Abhängig von der Tiefe des myometranen Defektes sollte nach Enukleation eine mehrschichtige Naht erfolgen ( $\sim$ Abb. 10). Ob eine 
Eröffnung des Cavum uteri einen weiteren Risikofaktor darstellt, wird in der Literatur kontrovers diskutiert. Eine Ruptur erfolgt meistens zwischen der 17. und 40. Schwangerschaftswoche. Gehäuft tritt dieses seltene Ereignis im frühen bis mittleren 3.Trimenon auf.

\section{PRAXISTIPP}

Bei guter Adaptation der Wundränder ist mit einer stabil geheilten Narbe nach 6 Monaten zu rechnen. Bis zu diesem Zeitpunkt sollte eine sichere Antikonzeption gewährleistet sein.

Der Entbindungsweg (spontan vs. primäre Sectio caesarea) muss je nach Lage und Ausdehnung des myometranen Defekts individuell mit der Patientin im „informed consent" besprochen werden.

\section{Radiofrequenzablation}

Die Radiofrequenzablation ist ein relativ neues Operationsverfahren. Es handelt sich um eine ultraschallgeführte Hochfrequenzablation. Entwickelt wurde dieses Verfahren, um Frauen eine zusätzliche minimalinvasive Option zur Behandlung von Myomen zu bieten, welche sicher, effektiv und vor allem uterusschonend ist. Der Mechanismus der Hochfrequenzablation ist bereits an einer Reihe anderer Körperstellen erforscht und etabliert, darunter Lunge, Leber, Niere und Brustdrüse. Die in einem Generator erzeugte Energie wird in Form eines Wechselstroms über eine im Myom platzierte Elektrode in Wärme umgewandelt.

\section{Merke}

Die bei der Radiofrequenzablation entstehenden Temperaturen von ca. $100^{\circ} \mathrm{C}$ führen zu einer Nekrose des behandelten Gewebes.

Die Form und die Größe der Ablationszone werden durch die gewählte Temperatur, Expositionszeit und Form der Ablationselektrode definiert. Postoperativ kommt es über Wochen bis Monate zu einer Größenreduktion der behandelten Myome. Sie verschwinden jedoch meist nicht komplett. Aufgrund der strukturellen Schädigung der Myome geht man aktuell davon aus, dass sie bei Eintritt einer Schwangerschaft kaum oder sogar gar nicht wachsen.

Aktuell stehen 2 verschiedene Verfahren zur Verfügung:

- transzervikale Radiofrequenzablation (Sonata, Gynesonics, Redwood, CA, USA)

- laparoskopische Radiofrequenzablation (Acessa Systems, Halt Medical, Brentwood, CA, USA)

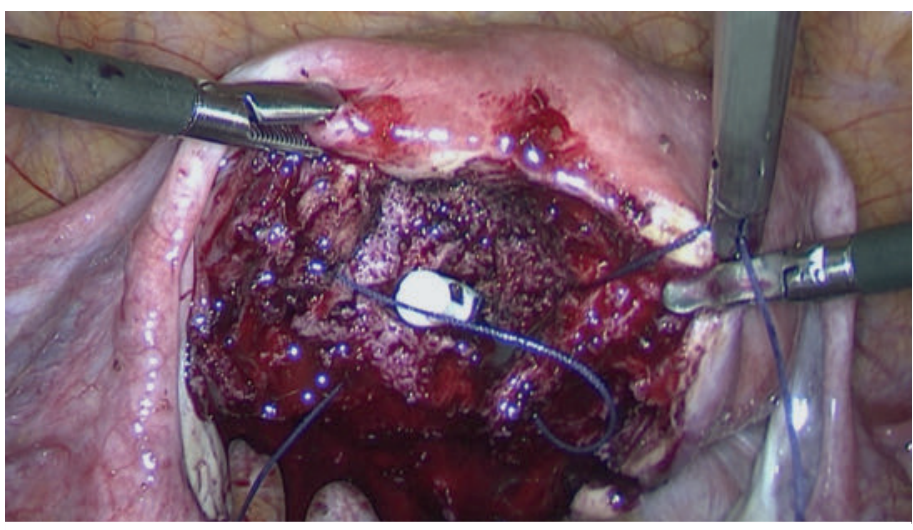

- Abb. 10 Laparoskopische tiefe Naht zur Rekonstruktion des Uterus bei Kavumeröffnung. Mittig ist das eröffnete Kavum mit liegendem Uterusmanipulator sichtbar.
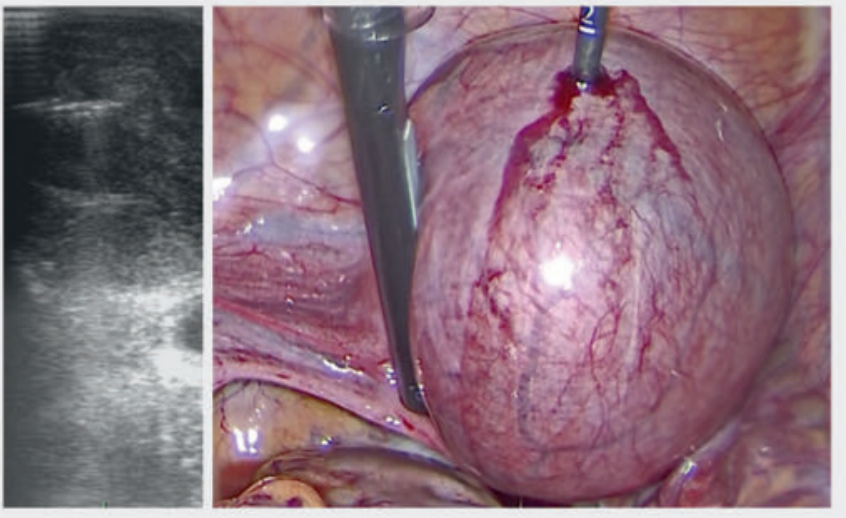

- Abb. 11 Laparoskopisch-sonografisch gesteuerte Radiofrequenzablation eines Fundusmyoms Typ 2-5. Links: sonografischer Live-Blick, Rechts: laparoskopischer Blick mit starrer Sonografiesonde (links) und platzierter, aktivierter Radiofrequenzsonde (rechts).

\section{Laparoskopische Radiofrequenzablation}

Dieser Eingriff wird im Rahmen einer Laparoskopie zur Therapie von intramuralen und subserösen Myomen durchgeführt. Über einen suprasymphysären 10-mmTrokar wird eine starre Ultraschallsonde in das Abdomen eingeführt. Sonografisch können nun alle Myome detektiert werden. Über eine weitere parallel zum Trokar platzierte 2-mm-Inzision wird zunächst mit einer ultraschallgeführten Feinnadelstanzbiopsie eine Gewebeprobe entnommen. Unter laparoskopisch-sonografischer Sicht werden die vorhandenen Myome abladiert ( $\mathbf{A} \mathbf{A b} \mathbf{b} . \mathbf{1 1}$ ). Wiederholte und überlagerte Ablationsschritte ermöglichen es, auch Myome größer als $4 \mathrm{~cm}$ sowie mehrere Myome sicher zu therapieren [13].

Vorteile dieses Verfahrens sind der geringe Blutverlust, kürzere Eingriffszeiten und ein kürzerer stationärer Auf- 
enthalt im Vergleich zur laparoskopischen Myomenukleation. Ein Schnitt im Myometrium ist nicht notwendig. Technikbedingt können gestielte Myome oder submuköse Myome nicht oder nicht suffizient therapiert werden.

Erste Fallberichte zeigen unauffällige Schwangerschaften mit termingerechten Lebendgeburten nach durchgeführter Therapie $(86,7 \%)$ mit gleichen Raten an vaginalen Entbindungen und Kaiserschnitten. In 13,3\% kam es zu einem Frühabort. Bei kleiner Fallzahl kam es nicht zu Frühgeburten oder Wachstumsrestriktionen, Uterusrupturen oder Plazentationsstörungen [14].

\section{Transzervikale Radiofrequenzablation}

Durch transzervikales Einführen der Ablationssonde mit integrierter abwinkelbarer Ultraschallsonde können intramurale und submuköse Myome therapiert werden. Eine histologische Sicherung der Myome kann bei diesem Verfahren nicht durchgeführt werden. Nach Platzierung der Radiofrequenzablationssonde im Myom wird die Ablationszone berechnet. Mit einer Sicherheitszone von ca. $1 \mathrm{~cm}$, welche intrauterin zu liegen kommen muss, wird das Myom unter sonografischer Kontrolle abladiert. Wiederholte und überlagerte Ablationsschritte ermöglichen es auch hier, Myome größer als $4 \mathrm{~cm}$ sowie mehrere Myome sicher zu therapieren. Auch bei diesem Verfahren können technikbedingt gestielte Myome nicht oder nicht suffizient therapiert werden.

Einzelne Fallberichte beschreiben unauffällige Schwangerschaften nach transzervikaler Radiofrequenzablation.

Für die Empfehlung der beiden Verfahren bei Frauen mit Kinderwunsch fehlt jedoch noch weitere Evidenz. Aktuell werden Studien zur Klärung der Auswirkungen der Radiofrequenzablation auf den Schwangerschaftsverlauf durchgeführt, die Datenlage ist aber begrenzt. Die vorliegenden Fallberichte über Schwangerschaftsverläufe mit Lebendgeburten sind jedoch vielversprechend.

\section{PRAXISTIPP}

Im Beratungsgespräch bezüglich der Verfahren zur Myomverkleinerung (operativ, interventionell und medikamentös) muss die Patientin darüber informiert werden, dass der Zeitraum der Myomverkleinerung bis zu 6 Monate postinterventionell andauern kann. Bei den postoperativen sonografischen Kontrollen sollte zur Beurteilung des Behandlungserfolges das Myomvolumen $(\mathrm{V})$ und nicht der Durchmesser (d) herangezogen werden, da der Durchmesser sich nicht so stark ändert wie das Volumen gemäß der Formel:

$\mathrm{V}=4 / 3 \cdot \pi \cdot(\mathrm{d} / 2)^{3}$

\section{Interventionelle Therapie}

Bei den interventionellen Therapieverfahren handelt es sich um minimal- oder nichtinvasive Therapieoptionen, die in radiologischen Abteilungen durchgeführt werden. Die beiden Therapieoptionen bilden eine Alternative zur gynäkologischen Operation der Myome sowie der medikamentösen Therapie.

Insbesondere bei Patientinnen mit Kontraindikationen oder Risikofaktoren für eine Operation können beide Verfahren eine Alternative darstellen.

\section{MRT-gesteuerter fokussierter Ultraschall (MRgFUS)}

Beim MRgFUS erzeugt man Magnetresonanztomografiekontrolliert mit fokussiertem Ultraschall Energie im Myom über einen „Brennglas-Effekt“. Es handelt sich hierbei um einen nichtinvasiven Eingriff ohne Schnitte, mit dem alle Myomtypen behandelt werden können. In vielen kleinen Schritten werden nacheinander Areale im Myom punktgenau auf $60-80^{\circ} \mathrm{C}$ erhitzt und so denaturiert. Durch diese kleinen und genau platzierten Schritte kann eine maximale Schonung des Endometriums erreicht werden. In den folgenden Wochen bis Monaten schrumpfen dann die Myome [15].

Mit einem eingeschränkten Behandlungserfolg muss bei mehr als 5 Myomen und großen Myomen über 10 Zentimetern gerechnet werden. Eine gute Durchblutung kann durch Kühlung des Zielvolumens zu einem verminderten Therapieansprechen führen. Darmüberlagerungen können den Schall so verändern, dass sie MRgFUS ineffektiv machen und den Darm schädigen. Narben und eine dicke subkutane Fettschicht sind ein Risikofaktor für Verbrennungen, da in diesen Geweben durch eine geringere Durchblutung eine erhöhte Gewebetemperatur entsteht [16].

Die Lebendgeburtenraten nach MRgFUS liegen bei $41 \%$ mit einer Abortrate von 28\% [17]. Das Verfahren ist zwar von der US-amerikanischen Arzneimittelbehörde FDA zur Myomtherapie bei Frauen mit Kinderwunsch zugelassen, wird in Deutschland bei fehlender Evidenz bezüglich ihrer Auswirkung in der Schwangerschaft aktuell nicht empfohlen [18].

\section{Embolisation der Arteria uterina (UAE)}

Bei der Embolisation der A. uterina handelt es sich um ein katheterbasiertes Verfahren, bei dem kleine Kunststoffpartikel über beide Äste der A. uterina in die das Myom versorgenden Gefäße injiziert werden. Der Eingriff erfolgt unter radiologischer Durchleuchtung. Bei optimaler Durchleuchtungszeit entsteht so eine Strahlenbelastung wie bei 2-3 Computertomografieuntersuchungen. Intramurale Myome bis zu einer Größe von $8 \mathrm{~cm}$ können behandelt werden. 


\section{FALLBEISPIEL}

Eine 27-jährige Nulligravida afrikanischer Herkunft stellt sich mit leichter Dysmenorrhö und Hypermenorrhö vor. Sie berichtet über seit Längerem bekannte Myome, die in letzter Zeit etwas größer geworden seien. Da nun ein aktueller Kinderwunsch bestehe, erfolgt die Vorstellung in der Myomsprechstunde. Im Rahmen der gynäkologischen Tastuntersuchung tastet der Uterus leicht vergrößert. Sonografisch zeigen sich 2 Myome. Ein Fundusmyom vom Typ 6 $(3,1 \times 3,2 \times 3,0 \mathrm{~cm})$ sowie ein Typ-6-Myom der Uterushinterwand $(0,7 \times 1,0 \times 0,6 \mathrm{~cm})(\triangleright$ Abb. 12).

Diskutiert werden das Myomwachstum in der Schwangerschaft und die Risiken einer Myomenukleation mit ggf. notwendiger primärer Sectio caesarea. Bei kleinem Myom (größter Durchmesser 3,5 cm), fehlender Kavumimpression sowie milder Symptomatik wird mit der Patientin zunächst die Spontankonzeption angestrebt. Bei ausbleibendem Schwangerschaftseintritt, Myomwachstum oder Beschwerdezunahme sollte die diagnostische Hysteroskopie mit laparoskopischer Myomenukleation und Chromopertubation empfohlen werden.
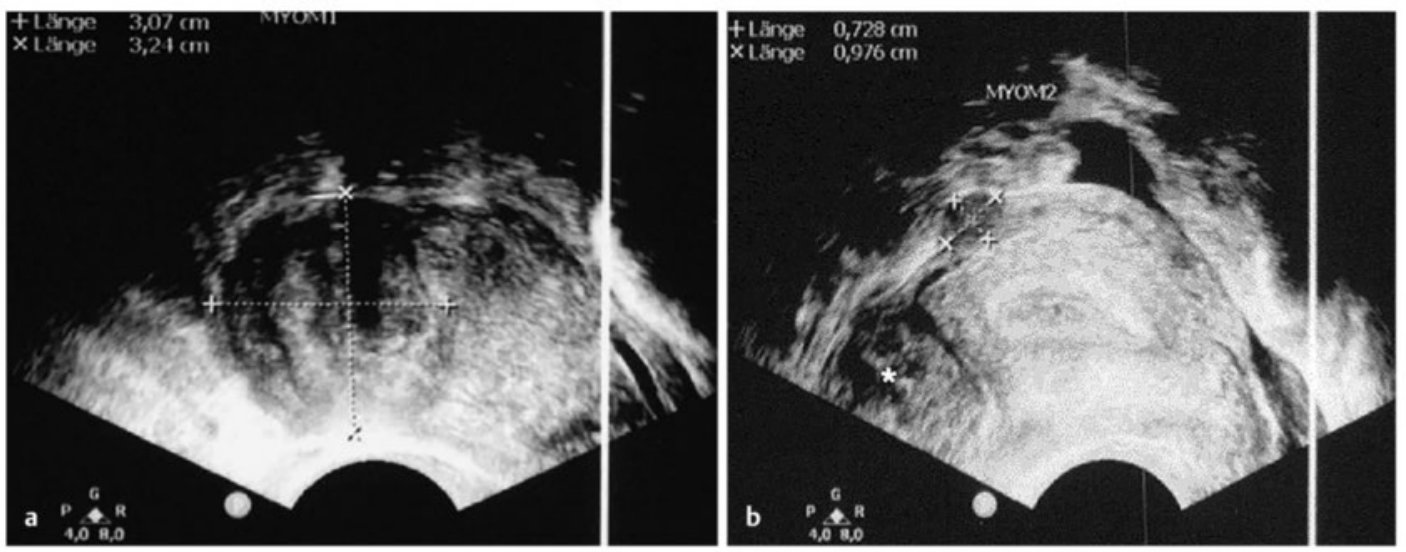

- Abb. 12 Sonografische Darstellung des Uterus: a Uterus längs mit 3,1 ×3,2 cm großem Fundusmyom in der linken Bildhälfte. b Uterus quer mit weiterem $1,0 \times 0,7 \mathrm{~cm}$ großem Hinterwandmyom. Das Fundusmyom ist im linken Bildanteil angeschnitten $\left({ }^{*}\right)$.

\section{PRAXISTIPP}

Der durch den Eingriff verursachte Gewebeinfarkt führt postoperativ meist zu starken Schmerzen, weshalb postinterventionell eine intensive, in der Regel intravenöse Schmerztherapie für 2-5 Tage notwendig ist.

Nach UAE kann man mit einer Reduktion der Myomgröße von $50-60 \%$ rechnen. Durch die induzierte Nekrose kommt es typischerweise zu einem bis zu 8 Wochen dauerndem Ausfluss. Auch kann es zu einem Ausstoß von Myomanteilen oder ganzen Myomen kommen. Dies kann in seltenen Fällen bis zu 50 Monate andauern [15].

Cave

Bei postinterventionellem Myomabgang und Infektionen liegt die Hysterektomierate bei bis zu $15 \%$.
Die Auswirkungen der UAE auf die Fertilität wird kontrovers diskutiert. Durch eine negative Beeinflussung der ovariellen Durchblutung kann es neben negativen Auswirkungen auf die endokrine Funktion zu einer Verschlechterung der Follikelentwicklung und Follikelreifung kommen. Auch werden negative Einflüsse auf die Implantation durch schlechtere Durchblutung des Endometriums beschrieben [15].

Karlsen beschreibt in seinem systematischen Review Schwangerschaftsraten von $50 \%$ in RCT, $51 \%$ und $69 \%$ in Kohortenstudien. Nach Myomenukleation lagen die Schwangerschaftsraten in den analysierten RCT bei 78\%. Auffällig ist jedoch die deutlich höhere Abortrate nach UAE (60 vs. 20\%) [19]. Zur besseren Beurteilung der Auswirkungen der UAE auf die Fertilität sind weitere Studien dringend notwendig.

Die UAE ist bei Frauen mit Kinderwunsch nicht kontraindiziert, sollte jedoch aufgrund der fehlenden Evidenz zur Auswirkung auf die Fertilität nicht die Therapie- 


\section{FALLBEISPIEL}

Es stellt sich eine 38-jährige Patientin mit bekanntem Uterus myomatosus und primärer Sterilität seit 4 Jahren vor. Anamnestisch liegt ein unauffälliges Spermiogramm des Partners vor. In der vaginalen bimanuellen Unterschung zeigt sich der Uterus bis 3 Querfinger über die Symphyse vergrößert. Im Douglas tastet sich ein derber Befund, der das kleine Becken fast komplett ausfüllt. Sonografisch lassen sich mindestens 4 intramurale Myome darstellen, davon ein Fundusmyom Typ 5 mit $5 \mathrm{~cm}$ Durchmesser und ein $8 \mathrm{~cm}$ messendes breit gestieltes Typ-7-Myom an der Hinterwand.

Es wurden die operative Hysteroskopie mit Myomresektion und die laparoskopische Myomenukleation inklusive Chromopertubation besprochen.

In der operativen Hysteroskopie zeigt sich ein normal konfiguriertes Kavum ohne Impression. Der Uterus ist um $45^{\circ}$ gegen den Uhrzeigersinn verdreht ( $\triangleright$ Abb. 13a). Laparoskopisch werden 3 Myome an der Vorderwand ( $1 \times$ Typ 6, $2 \times$ Typ 7), ein breit gestieltes Myom an der Hinterwand (Typ 7), sowie ein Typ-6- und ein Typ-5-Myom an der Hinterwand enukleiert ( $\bullet$ Abb. 13 b-d). An der Hinterwand kommt es zur Kavumeröffnung. Insgesamt werden Myome mit einem Gewicht von $350 \mathrm{~g}$ entfernt. Der Uterus wird mit zweischichtigen Nähten rekonstruiert und erscheint im Anschluss normal groß ( $\bullet$ Abb. 13e). Nach 2 Nächten stationärer Observatio wird die Patientin entlassen. Eine sichere Antikonzeption für 6 Monate sowie eine primäre Sectio caesarea bei Eintritt einer Schwangerschaft werden empfohlen.
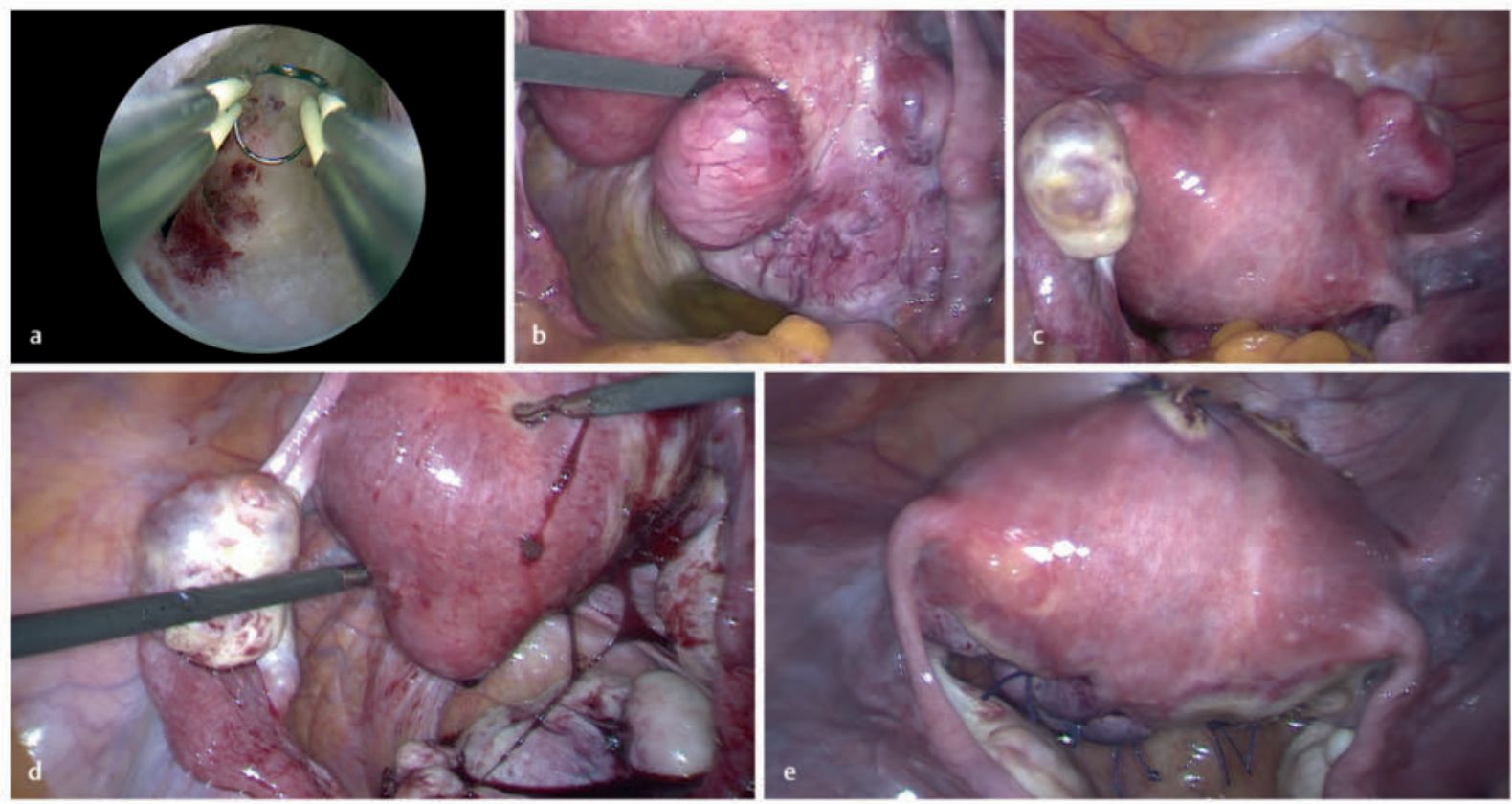

- Abb. 13 Intraoperative Bilddokumentation a Blick in das Cavum uteri, 45 gegen den Uhrzeigersinn verdreht. b Hinterwandmyom Typ 7 (re.) und Typ 6 (li.) c Verdrehter Uterus mit Myomen an der Vorderwand (Typ 6 und 7). d Großes Hinterwandmyom Typ 5, welches nach Entfernung des Typ-7-Myoms an der rechten Hinterwand sichtbar wurde. Im Douglas befinden sich bereits entfernte Myome. e Abschlussbild: Uterus nach Enukleation aller Myome und Uterusrekonstruktion.

methode der ersten Wahl darstellen. Bei Patientinnen mit großen, schwer zugänglichen oder multiplen Myomen kann die UAE jedoch, nach ausführlicher Aufklärung über die unklaren Auswirkungen auf die Fertilität, eine Therapieoption darstellen.

\section{Medikamentöse Therapie}

GnRH-Analoga

Unter GnRH-Analoga verstehen sich ähnliche (analoge) chemische Verbindungen wie Gonadotropin-Releasing-
Hormon (GnRH), die mit hoher Affinität an die GnRH-Rezeptoren der Hypophyse binden. Bei Therapie über mehrere Wochen resultiert eine Downregulation der hypophysären GnRH-Rezeptoren. Durch das Absinken der LHund FSH-Werte kommt es zum Absinken des Östrogenspiegels und der Follikelreifung.

Eine GnRH-Analoga-Therapie vermag in 3-6 Monaten das Myomvolumen um 50-60\% zu vermindern. Meist tritt nach 2-4 Wochen aufgrund des erniedrigten Östrogen- 
spiegels eine Amenorrhö ein. Die typischen Nebenwirkungen einer $\mathrm{GnRH}$-Analoga-Therapie sind die eines „künstlichen Klimakteriums“. Unter anderem kommt es zu einer Knochendemineralisierung, Hitzewallungen, Reduktion der Libido und trockenen Schleimhäuten. Bei einer Therapie, die länger als 3 Monate geplant ist, sollte deshalb eine (parallele) „Add-back“-Therapie mit einem niedrigdosierten Östrogen erfolgen.

Cave

Nach Absetzen der Medikation kommt es meist nach einigen Monaten erneut zu einem Myomwachstum.

Sollte nach der Therapie eine Myomenukleation geplant sein, so kann diese durch die hierdurch weicheren Myome unter Umständen erschwert werden.

Aufgrund des Nebenwirkungsprofil von GnRH-Analoga spielen diese heutzutage in der klinischen Routine kaum mehr eine Rolle. Sollte dennoch eine Therapie geplant werden, so sollten die Nebenwirkungen und das wahrscheinlich resultierende Myomwachstum nach Absetzen der Medikation ausführlich mit der Patientin besprochen werden.

\section{Ulipristalacetat (Esmya ${ }^{\circledR}$ )}

Bei Ulipristalacetat (UPA) handelt es sich um einen selektiven Progesteronrezeptor-Modulator (SPRM), der 2012 in der Europäischen Union zur Behandlung von symptomatischen Myomen zugelassen wurde. Die Behandlung erfolgt durch die Einnahme einer Tablette $5 \mathrm{mg}$ UPA pro Tag für 3 Monate. In klinischen Studien (PEARL 1-4) konnte eine schnelle und effektive Blutungskontrolle und Reduktion des Myomvolumens in bis zu $80 \%$ der Fälle gezeigt werden [20]. Ende 2017 kam es zu einem vorläufigen Verschreibungsstopp aufgrund eines Risikobewertungsverfahrens, da in 8 Fällen ein schwerer Leberschaden unter Therapie mit UPA aufgetreten war. Bei 4 Patientinnen war in der Folge eine Lebertransplantation notwendig, eine Patientin verstarb an einer Sepsis.

Mitte 2019 war UPA nach positivem Risikobewertungsverfahren erneut auf dem europäischen Markt verfügbar. Voraussetzung für eine Therapie war nunmehr ein unauffälliger GOT- und GPT-Wert vor Therapiebeginn. Die Leberwerte mussten $1 \times$ pro Monat kontrolliert werden. Bei Werten, die mehr als der 3-fachen Norm entsprachen, musste die Therapie abgebrochen werden. Bestehende Leberfunktionsstörungen wurden als Kontraindikation in den Beipackzettel aufgenommen.

Eine Cochrane-Analyse von 2017 beschreibt eine ähnlich gute Wirkung in Bezug auf eine Myomverkleinerung, myomassoziierte Blutungen und eine Erhöhung des Hämoglobin-Levels [20]. Unter Therapie sind nicht physiologische Veränderungen des Endometriums bei bis zu 2/3 al- ler behandelten Patientinnen beschrieben. Diese sind meist nach 6 Monaten nicht mehr nachweisbar.

UPA zeichnet sich durch eine bessere Verträglichkeit aus im Vergleich zu einer Therapie mit GnRH-Analoga mit konsekutiver Senkung des Östrogenspiegels und hierdurch provozierten Wechseljahresbeschwerden. Durch UPA kommt es zu einer Hemmung der Ovulation. UPA ist jedoch nicht zur Antikonzeption zugelassen, sodass während der Einnahme eine sichere Verhütung mittels Barrieremethode empfohlen wird.

In der Literatur sind inzwischen 71 Schwangerschaften unter oder nach UPA-Therapie beschrieben. Fünf Schwangerschaften traten während der UPA-Einnahme auf. Hieraus resultierten 5 Lebendgeburten, ein Abort und eine Interruptio [21]. Bei alleiniger Therapie mit UPA ohne anschließende Myomenukleation zeigten sich Schwangerschaftsraten von bis zu $71 \%$ mit $2 / 3$ Lebendgeburten und $1 / 3$ Aborten [22].

Im März 2020 wurde nach einem erneuten schweren Leberschaden unter Einnahme von UPA mit regelmäßigen Leberwertkontrollen ein erneutes Risikobewertungsverfahren eingeleitet. Anfang September 2020 bewertete der Ausschuss für Risikobewertung im Bereich der Pharmakovigilanz (PRAC) der EMA das Nutzen-Risiko-Verhältnis von UPA $5 \mathrm{mg}$ als nicht günstig und empfahl den Widerruf der Zulassung. Die Anwendung von UPA zur postkoitalen Therapie bleibt hiervon unangestastet.

\section{KERNAUSSAGEN}

- Myome sind gutartige hormonsensible Tumoren der glatten Muskulatur des Uterus, die bei den meisten Patientinnen keine Symptome hervorrufen.

- Insbesondere submuköse und intramurale Myome, welche die Form des Kavums verändern, können die Fertilität negativ beeinflussen.

- Der Goldstandard zur Diagnostik ist die bimanuelle Untersuchung mit transvaginalem Ultraschall.

- Einer spezifischen Therapie sollten nur Frauen mit Beschwerden oder den Kinderwunsch negativ beeinflussenden Myomen zugeführt werden. Der Goldstandard ist hierfür immer noch die operative Entfernung der ursächlichen Myome per Hysteroskopie und/oder Laparoskopie. Nur in seltenen, ausgedehnten Fällen ist die offen-chirurgische Entfernung notwendig.

- Erfolgversprechende operative und interventionelle Therapieoptionen stehen ebenfalls zur Verfügung. Diese sind bisher jedoch noch nicht ausreichend bezüglich ihres Nutzens und des Einflusses auf eine Schwangerschaft untersucht. Sie können im Individualfall aber eine sehr gute Behandlungsoption darstellen.

- Die Aufgabe des untersuchenden und beratenden Arztes ist es, die für die jeweilige Patientin passende Therapie herauszuarbeiten. 
Interessenkonflikt

Erklärung zu finanziellen Interessen

Forschungsförderung erhalten: nein; Honorar/geldwerten Vorteil für Referententätigkeit erhalten: ja, von einer anderen Institution (Pharma- oder Medizintechnikfirma usw.); Bezahlter Berater/interner Schulungsreferent/Gehaltsempfänger: nein; Patent/Geschäftsanteile/Aktien (Autor/Partner, Ehepartner, Kinder) an im Bereich der Medizin aktiven Firma: nein; Patent/Geschäftsanteile/Aktien (Autor/Partner, Ehepartner, Kinder) an zu Sponsoren dieser Fortbildung bzw. durch die Fortbildung in ihren Geschäftsinteressen berührten Firma: nein.

Erklärung zu nichtfinanziellen Interessen

Die Autorinnen/Autoren geben an, dass kein Interessenkonflikt besteht.

Autorinnen/Autoren

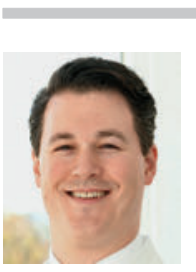

\section{Felix Neis}

Dr. med. 2001-2008 Studium der Humanmedizin an der Ruprecht Karls Universität Heidelberg. 2008-2014 Facharztausbildung Gynäkologie und Geburtshilfe. 2014 Facharzt Gynäkologie und Geburtshilfe. Seit 2017 Leiter Myomzentrum am Department für Frauengesundheit der Universität Tübingen. 2019 Stellvertretender Leitender Oberarzt am Department für Frauengesundheit der Universität Tübingen. Schwerpunkte: minimalinvasive Chirurgie, Therapie benigner und maligner Uteruserkrankungen, Dysplasie des weiblichen Genitales.

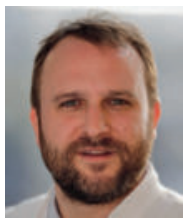

\section{Christoph Oettling}

Dr. med. 2005-2012 Studium der Humanmedizin an der Eberhard Karls Universität Tübingen. 2013-2019 Facharztausbildung Gynäkologie und Geburtshilfe. 2014 Facharzt Gynäkologie und Geburtshilfe. Seit 2019 Oberarzt am Department für Frauengesundheit der Universität Tübingen. Schwerpunkte: Urogynäkologie, Endometriose.

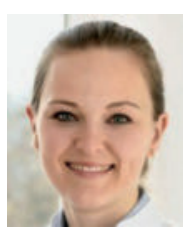

\section{Katrin Neis}

2008-2014 Studium der Humanmedizin an der Eberhard Karls Universität Tübingen. 2014-2020 Facharztausbildung Gynäkologie und Geburtshilfe. 2020 Fachärztin Gynäkologie und Geburtshilfe. Seit 2020 Fachärztin am Department für Frauengesundheit der Universität Tübingen. Schwerpunkte: Onkologie, Dysplasie des weiblichen Genitales, Myome, Endometriose.

\section{Korrespondenzadresse}

\section{Dr. med. Felix Neis}

Department für Frauengesundheit

Universität Tübingen

Calwerstraße 7

72076 Tübingen

Deutschland

felix.neis@med.uni-tuebingen.de
Wissenschaftlich verantwortlich gemäß Zertifizierungsbestimmungen

Wissenschaftlich verantwortlich gemäß Zertifizierungsbestimmungen für diesen Beitrag ist Dr. med. Felix Neis, Tübingen.

Literatur

[1] Ahrendt H], Tylkoski H, Rabe T et al. Prevalence of uterine myomas in women in Germany: data of an epidemiological study. Arch Gynecol Obstet 2016; 293: 1243-1253

[2] Downes E, Sikirica V, Gilabert-Estelles J et al. The burden of uterine fibroids in five European countries. Eur J Obstet Gynecol Reprod Biol 2010; 152: 96-102

[3] Donnez J, Dolmans MM. Uterine fibroid management: from the present to the future. Hum Reprod Update 2016; 22: 665-686

[4] Ciavattini A, Di Giuseppe J, Stortoni P et al. Uterine fibroids: pathogenesis and interactions with endometrium and endomyometrial junction. Obstet Gynecol Int 2013; 2013: 173184

[5] Munro MG et al., FIGO wWorking Group on Menstrual Disorders. FIGO classification system (PALM-COEIN) for causes of abnormal uterine bleeding in non-gravid women of reproductive age. Int J Gynaecol Obstet 2011; 113: 3-13

[6] Denschlag D et al. DGGG. S2k-Leitlinie: Uterine Sarkome. 2018. AWMF-Leitlinienprogramm DGGG, SGGG, OEGGG. Im Internet (Stand: 01.10.2020): https://www.awmf.org/leitlinien/detail/II/015-074.html

[7] Metwally M, Raybould G, Cheong YC et al. Surgical treatment of fibroids for subfertility. Cochrane Database Syst Rev 2020; (1): CD003857

[8] Pritts EA, Parker WH, Olive DL. Fibroids and infertility: an updated systematic review of the evidence. Fertil Steril 2009; 91: $1215-1223$

[9] Practice Committee of the American Society for Reproductive Medicine. Removal of myomas in asymptomatic patients to improve fertility and/or reduce miscarriage rate: a guideline. Fertil Steril 2017; 108: 416-425

[10] Vitagliano A, Noventa M, Di Spiezio Sardo A et al. Uterine fibroid size modifications during pregnancy and puerperium: evidence from the first systematic review of literature. Arch Gynecol Obstet 2018; 297: 823-835

[11] Kongnyuy E], Wiysonge CS. Interventions to reduce haemorrhage during myomectomy for fibroids. Cochrane Database Syst Rev 2009; (3): CD005355

[12] Cezar C, Becker S, Di Spiezio Sardo A et al. Laparoscopy or laparotomy as the way of entrance in myoma enucleation. Arch Gynecol Obstet 2017; 296: 709-720

[13] Grube M, Neis F, Brucker SY et al. Uterine fibroids - Current trends and strategies. Surg Technol Int 2019; 34: 257-263

[14] Berman JM, Shashoua A, Olson C et al. Case series of reproductive outcomes after laparoscopic radiofrequency ablation of symptomatic myomas. J Minim Invasive Gynecol 2020; 27: 639-645

[15] David M, Kröncke T. UAE und MRgFUS zur nichtoperativen Myomtherapie - ein Update. Frauenarzt 2019; 5

[16] Duc NM, Keserci B. Review of influential clinical factors in reducing the risk of unsuccessful MRI-guided HIFU treatment outcome of uterine fibroids. Diagn Interv Radiol 2018; 24: 283-291 
[17] Masciocchi C, Arrigoni F, Ferrari F et al. Uterine fibroid therapy using interventional radiology mini-invasive treatments: current perspective. Med Oncol 2017; 34: 52

[18] Kröncke T, David M. MR-guided focused ultrasound in fibroid treatment - Results of the 4th Radiological-Gynecological Expert Meeting. Geburtshilfe Frauenheilkd 2019; 79: 693-696

[19] Karlsen K, Hrobjartsson A, Korsholm M et al. Fertility after uterine artery embolization of fibroids: a systematic review. Arch Gynecol Obstet 2018; 297: 13-25

[20] Lethaby A, Puscasiu L, Vollenhoven B. Preoperative medical therapy before surgery for uterine fibroids. Cochrane Database Syst Rev 2017; (11): CD000547

[21] Donnez J, Arriagada P, Donnez O et al. Emerging treatment options for uterine fibroids. Expert Opin Emerg Drugs 2018; 23: $17-23$
[22] Luyckx M, Squifflet JL, Jadoul P et al. First series of 18 pregnancies after ulipristal acetate treatment for uterine fibroids. Fertil Steril 2014; 102: 1404-1409

Bibliografie

Frauenheilkunde up2date 2020; 14: 579-595

DOI 10.1055/a-1065-8028

ISSN $1439-3719$

(c) 2020. Thieme. All rights reserved.

Georg Thieme Verlag KG, Rüdigerstraße 14,

70469 Stuttgart, Germany 


\section{Punkte sammeln auf CME.thiemede}

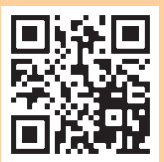

Diese Fortbildungseinheit ist in der Regel 12 Monate online für die Teilnahme verfügbar.

Den genauen Einsendeschluss finden Sie unter https://eref.thieme.de/CXE97SI.

Sollten Sie Fragen zur Online-Teilnahme haben, finden Sie unter https://cme.thieme.de/hilfe

eine ausführliche Anleitung. Wir wünschen viel Erfolg beim Beantworten

der Fragen!

Unter https://eref.thieme.de/CXE97SI oder über den QR-Code kommen Sie direkt zur Startseite des Wissenstests.

VNR 2760512020158723813

\section{Frage 1}

Welche Aussage zur Inzidenz und Entstehung von Myomen ist richtig?

A Im gebärfähigen Alter sind ca. $60 \%$ der Frauen von Myomen betroffen.

B Die meisten Myome wachsen in der Postmenopause.

C Myome sind gutartige Tumoren des Uterus und bestehen aus glatter Muskulatur.

D Genetische Faktoren gehören nicht zu den Ursachen der Myomentstehung.

E Durch die Einnahme von Hormonen lässt sich das Wachstum von Myomen hemmen.

\section{Frage 2}

Symptome und Einteilung der Myome: Welche Aussage ist richtig?

A Die Fertilität wird von der Lage der Myome gleichmäßig negativ beeinflusst.

B Die Größe des Myoms hat keinen Einfluss auf die Fertilität.

C Die Typen 0 bis 2 beschreiben subseröse Myome.

D Submuköse Myome können eine Implantation negativ beeinflussen.

E Unterbauchschmerzen werden in der Regel nur von intramuralen Myomen hervorgerufen.

\section{Frage 3}

Welche Aussage zur Diagnostik von Myomen ist falsch?

A Durch die bimanuelle Untersuchung können die Größe des Uterus und die Lage von Myomen bestimmt werden.

B Bei Verdacht auf Myome kann immer auf die rektovaginale Untersuchung verzichtet werden.

C Der transvaginale Ultraschall ist der Goldstandard zur Darstellung der Myome.

D Die Klassifikation der Myome nach der FIGO-Klassifikation hat sich wegen der guten Reproduzierbarkeit durchgesetzt.

$\mathrm{E}$ Myome sollten immer mit Durchmessern in mindestens 2 Ebenen beschrieben werden.

\section{Frage 4}

Welche Aussage zur Diagnostik von Myomen ist richtig?

A Zur Abschätzung der Malignität eines Myoms hat sich die Bestimmung des FGF1 (fibroid growth factor 1 ) als hilfreich erwiesen.

B Bei großen Myomen sollte auch an einen Nierenultraschall gedacht werden.

C Durch MRT und CT kann ein Sarkom sicher ausgeschlossen werden.

D Bei Verwendung der FIGO-Klassifikation kann auf die Lagebeschreibung (Fundus, Vorderwand etc.) verzichtet werden.

E Bei Verdacht auf Myome kann auf die Untersuchung mit Spekula verzichtet werden.

\section{Frage 5}

Welche Aussage ist richtig?

A Intramurale Myome verschlechtern die Fertilität am stärksten.

B Bei Veränderungen des Kavums ist die Fertilitätschance verschlechtert.

C Die Lage der Myome ist in Bezug auf die Fertilität irrelevant.

D Unabhängig von der Lage können Myome unter $3 \mathrm{~cm}$ immer belassen werden.

E Vorderwandmyome stören die Fertilität am meisten.

\section{Frage 6}

Welche Frage zu Fertilität und Schwangerschaft bei Vorliegen von Myomen ist falsch?

A Myome wachsen am stärksten im 1. Trimenon.

B Die Myomgröße ist im Wochenbett regredient.

C Circa 70\% aller Schwangerschaften mit Myomen verlaufen unauffällig.

D Das Myomwachstum bei subserösen Myomen ist in der Schwangerschaft am ungefährlichsten.

E Im 3. Trimenon kommt es in der Regel zu einem weiten Wachstumsschub.

- Weitere Fragen auf der folgenden Seite... 


\title{
Punkte sammeln auf CMIE.thieme.de
}

\author{
Fortsetzung ...
}

\section{Frage 7}

Welcher der folgenden Aspekte sollte in der Beratung über eine Myomtherapie keinen Einfluss haben:
A Versicherungsstatus
B Alter der Patientin
C Aktualität des Kinderwunsches
D Myomcharakteristika
E Patientenwunsch

\section{Frage 8}

Welche Aussage zur operativen Hysteroskopie ist falsch?

A Die Domäne der operativen Hysteroskopie sind Typ-0- bis Typ-2-Myome.

B Die operative Hysteroskopie sollte in der 1. Zyklushälfte erfolgen.

C Ziel ist die Resektion der größten intrakavitären Myome.

D Die Patientin sollte über die Möglichkeit einer zweizeitigen hysteroskopischen Myomresektion informiert werden.

E Nach hysteroskopischer Myomresektion kann in der Regel ein Spontanpartus bei Eintritt einer Schwangerschaft angestrebt werden.

\section{Frage 9}

Welche Aussage zur laparoskopischen Myomenukleation ist falsch?

A Bei Kavumeröffnung sollte eine mehrschichtige Naht erfolgen.

B Bei ausgedehntem Myombefall kann das abdominale Vorgehen sicherer sein.

C Adhäsionen nach Myomenukleation entstehen meistens an der Vorderwand.

D Rezidive entstehen bei bis zu 60\% der Frauen innerhalb von 5 Jahren.

E Durch Verhinderung von Hämatomen und Nekrosen kann das Risiko einer Uterusruptur gesenkt werden.

\section{Frage 10}

Welche Aussage zur Therapie von Myomen ist richtig?

A Bei der laparoskopischen Radiofrequenzablation kann bei guter Sicht auf die intraoperative Ultraschallkontrolle verzichtet werden.

B Gestielte Myome können mit dem MRgFUS gut behandelt werden.

C Die UAE wird besonders bei Seitenwandmyomen $>5 \mathrm{~cm}$ empfohlen.

D Es gibt in Deutschland aktuell keine Empfehlung zur UAE und dem MRgFUS bei Patientinnen mit Kinderwunsch.

E Risikopatientinnen für abdominale Operationen sind Ausschlusskriterien für MRgFUS, UAE und transzervikale Radiofrequenzablation. 